$>$ Au début du mois d'août 1848, six moines augustins du monastère Saint-Thomas de Brünn (Moravie) envoient une pétition à l'Assemblée impériale constituante de Vienne. La signature de Gregor Mendel au bas de ce texte en fait un des rares documents susceptibles de nous informer sur son comportement durant ce qui fut pour lui une période cruciale.

\section{8 Gregor Mendel, le moine qui voulait être citoyen}

\section{Christiane Nivet}

La vie personnelle de Gregor Johann Mendel (18221884) est mal connue car on ne dispose pas de ses papiers personnels, détruits après son décès. Elle a été reconstituée à partir de documents officiels, conservés soit dans le monastère Saint-Thomas de Brünn (actuellement Brno) où il vécut de 1843 à 1884 , soit dans les archives de l'Administration impériale viennoise ou dans celles de l'Administration locale de Moravie, alors province d'Empire des Habsbourg. Deux biographies de Mendel existent, écrites par des Moraves, la première, parue en 1924 en allemand, a pour auteur Hugo Iltis [1] qui a pu rencontrer des contemporains de Mendel; la seconde, publiée par Vitezlav Orel, est parue en anglais en 1996 [2]. Ni l'une ni l'autre, à notre avis, ne réussit pleinement à capter la personnalité du moine Mendel, notamment telle qu'elle s'est exprimée pendant la période révolutionnaire de 1848 .

\section{La pétition de 1848}

Gregor Mendel, célèbre pour ses travaux sur l'hérédité, s'engagea aussi en politique, comme en témoigne la pétition, signée en août 1848 par six moines augustins de son monastère, qui fut envoyée à l'Assemblée constituante impériale viennoise. Elle témoigne de la participation des moines au mouvement revendicatif de 1848. La signature de Mendel au bas de ce texte et le fait qu'il aurait été rédigé de sa main - d'après Weiling [3], un autre de ses biographes - font supposer que Mendel lui-même en était l'auteur. Ce manuscrit permet de connaître la façon dont Mendel a perçu les événements au cours de cette période d'agitation et son

Article reçu le 26 août 2005, accepté le 23 décembre 2005.

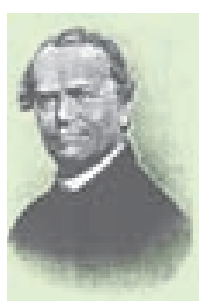

Université Paris7. nivet@paris7.jussieu.fr

attitude vis-à-vis de son monastère et de la hiérarchie religieuse. Nous présentons ici (en italiques), par paragraphes numérotés de 1 à 6 , la traduction française intégrale du texte qui a été retrouvé en 1955 dans les archives relatives aux années 1848-49 de l'Assemblée constituante. En voici le début:

1. «Haute Assemblée de l'Empire, À l'occasion de la réorganisation politique de notre patrie, les soussignés estiment de leur devoir, au nom de l'humanité, d'attirer l'attention de la Haute Chambre autrichienne sur le fait que toute une classe d'hommes, non négligeable, dans les États autrichiens, totalement exclue des conquêtes de mars et de mai, ne peut partager qu'avec amertume la liesse des peuples qui se sentent comblés 》.

Le contenu de cette pétition est étroitement lié aux événements révolutionnaires de mars 1848 qui, de Vienne, ont gagné les provinces sous la domination des Habsbourg.

\section{Contexte révolutionnaire de la pétition}

Le 13 mars 1848, à Vienne, un mouvement revendicatif mené par des intellectuels enseignants et des étudiants entraîna la démission du ministre Metternich, suivie d'un revirement libéral de l'empereur Ferdinand (1835-1848) qui promit la rédaction d'une constitution («conquêtes de mars»). Simultanément, une vague de ferveur révolutionnaire se répandit dans l'Empire autrichien. La participation des moines augustins de Saint-Thomas à Brno fut précoce car, enseignants euxmêmes, ils se rallièrent au mouvement dès le 17 mars. Les pères Bratanek et Gabriel se joignirent à une dépu- 
tation d'étudiants de Brno en soutien au mouvement amorcé à Vienne, tandis que le supérieur Napp recevait au monastère un groupe d'étudiants viennois. Le lendemain, il célébra en l'honneur des étudiants tués par l'armée au cours des échauffourées de Vienne, une messe pendant laquelle le père Krizkovsky dirigea le Requiem de Cherubini et le père Klacel fit un sermon où il demandait l'abolition du travail féodal et l'amélioration des conditions de vie des travailleurs [2].

Ces moines militaient aussi en faveur de la résurrection de la nation tchèque, contrairement à la bourgeoisie locale d'origine allemande et conservatrice. Ils furent, avec leurs amis professeurs laïcs, parmi les meneurs de la rébellion à Brno. L'abbaye étant un gros propriétaire terrien, l'abbé Napp siégeait de droit à la Diète provinciale et, lorsqu'il fut question d'organiser les élections de la nouvelle Diète, il s'opposa à l'augmentation de la représentation des bourgeois et favorisa une représentation proportionnelle des régions rurales. La nouvelle Diète morave se réunit à Brno le 31 mai et, à l'instigation de Hans Kudlich, un paysan originaire de Silésie comme Mendel, proclama l'abolition du servage en Moravie le 11 juillet. Les travaux de la Diète s'interrompirent lorsque le gouvernement de Vienne accepta enfin le principe d'une assemblée constituante élue au suffrage universel : ce sont les «conquêtes de mai» dont parle la pétition. D'où «la liesse des peuples qui se sentent comblés », car cette assemblée de 383 membres élus - dont 38 pour la Moravie et 10 pour la Silésie - fut composée de représentants venant de diverses provinces de l'Empire.

Ce parlement, réuni à Vienne le 22 juillet 1848, devait rédiger la nouvelle constitution et formuler les conditions pour sortir du féodalisme. Le début d'août 1848 est une période faste: tous croient en cette Haute Assemblée de l'Empire et attendent d'elle «la réorganisation politique de leur patrie », en accord avec les revendications des mois précédents. De nombreuses pétitions furent alors envoyées au parlement parmi lesquelles celle qui nous intéresse. Daté du 8 août 1848 , le document a été enregistré le 11 et communiqué le 16 au comité réuni pour la nouvelle constitution.

\section{Signataires et objectif de la pétition}

Les signataires se présentent comme des «professeurs et chargés d'âmes, prêtres de l'abbaye des Augustins SaintThomas à Alt-Brünn, (qui) se permettent d'exprimer à la Haute Assemblée de l'Empire la juste requête de la reconnaissance de leur droit constitutionnel de citoyens » et terminent en demandant «qu'il leur soit permis, dans la mesure de leurs aptitudes et du mérite acquis par le passé, de consacrer toute leur énergie spirituelle à l'enseignement public ».
En tête des signataires se trouve le père Mattheus Klacel, alors âgé de 40 ans, brillant botaniste et ex-professeur de philosophie, déchargé de ses cours par l'évêque de Brünn à cause de ses positions pro-hégéliennes en 1843, époque où Mendel est entré au monastère. Klacel, devenu l'ami et le mentor en sciences naturelles de Mendel, était de plus un fervent militant de la renaissance nationale tchèque. Vient ensuite la signature du père Gabriel, 37 ans, professeur de mathématiques à Brünn, qui a participé directement aux événements. Sont également signataires, deux moines, J. Lindenthal, 38 ans, vicaire de la paroisse d'Alt-Brünn, et Benedict Fogler, 36 ans, professeur de français et d'italien, qui seront plus tard les collaborateurs de Mendel au cours de son expérimentation sur les pois. Autre signataire, Chrysostomus Cyganek, jeune clerc de 23 ans entré à Saint-Thomas la même année que Mendel, trop jeune pour être prêtre, a mis son nom sous celui de Lindenthal avec la mention «candidat professeur». Enfin, vient en dernier, accompagnée de la même mention, la signature de Gregor Mendel, 26 ans, prêtre et vicaire de la paroisse d'Alt-Brünn.

Lindenthal et Cyganek partagent avec Mendel le déplaisir d'avoir hérité inopinément de la charge de la paroisse d'Alt-Brünn. La pétition demande «la reconnaissance de leur droit constitutionnel de citoyens » et la possibilité d'exercer en tant que professeurs. Klacel souhaite retrouver un poste d'enseignant en philosophie; Mendel, Lindenthal et Cyganek veulent devenir professeurs, revendication que soutiennent les signataires déjà enseignants.

\section{Auteur de cette pétition}

Mendel, ayant vocation pour le professorat, a mené ses études secondaires dans des conditions misérables. Ne supportant plus les privations, il est entré à Saint-Thomas en 1843 dans l'espoir qu'à l'instar d'autres moines il serait envoyé à l'université de Vienne aux frais du monastère, pour y acquérir les diplômes nécessaires à sa qualification d'enseignant. Dans ce but, il accepta de suivre cinq longues années d'études en théologie pour devenir prêtre et ne ménagea pas sa peine pour y parvenir. Mais en 1847 , trois moines responsables de la paroisse d'Alt-Brünn décédèrent subitement [1]. À la demande de son abbé, par dispense, car il n'avait pas achevé ses études, Mendel fut nommé sous-diacre le 22 juillet, jour de ses vingt-cinq ans (âge requis pour devenir prêtre) et, dans l'urgence, diacre le 24, enfin ordonné prêtre le 6 août, afin de pourvoir au service paroissial. L'année suivante, il termine sa formation religieuse fin juin et se prépare à entrer à l'Université. Mais hélas, le 22 juillet 1848, l'évêque le nomme curé d’Alt-Brünn, ce qui sonne le glas de ses espérances universitaires et professorales. Ironie du sort, ce jour anniversaire de ses 26 ans est aussi celui de l'ouverture de l'Assemblée Impériale Constituante!

Mendel constate avec amertume qu'il ne sera jamais professeur et que son état de prêtre régulier l'assujettit à une sorte de féodalité religieuse pour le reste de sa vie, ce qui le conduit à écrire cette pétition datée du 8 août. D'emblée, en termes véhéments, elle décrit précisément la condition du prêtre régulier, totalement dépourvu de droits civiques: 
2. «D’après le droit civil autrichien, le religieux régulier se trouve derrière le criminel-dont les droits civiques ne sont que suspendus - comme un être civiquement privé de droits; les droits du citoyen autrichien lui sont refusés, il est selon le texte juridique déclaré civiquement mort. Comme c'est le cas pour l'aliéné, l'enfant et la personne placée sous curatelle, tout contrat qu'il conclut est nul; il n'a le droit ni de se porter caution, ni de porter témoignage devant un tribunal, ni d'être témoin ou assistant lors d'un mariage, ni de tenir le rôle de parrain lors d'un baptême ou d'une confirmation; il n'a le droit ni d'hériter, ni de léguer à sa famille, souvent dans le besoin, le peu de bien souvent durement acquis; juridiquement, seul son ordre hérite de lui et pour lui. C'est ainsi que, jusqu'à maintenant, les statuts civiques de la monarchie absolue se prononcent en faveur des communautés religieuses, mais au préjudice délibéré du religieux pris individuellement qui malheureusement n'en a saisi la signification pratique, lourde de contenu, qu'après des années, au prix de tristes expériences. Ces lois sont encore, depuis le 13 mars, pleinement en vigueur, elles n'ont pas été suspendues et jusqu'à aujourd'hui le droit de la citoyenneté constitutionnelle n'a pas été accordé au religieux régulier. »

Entouré de moines - rebelles quand il s'agissait de défendre le droit et les libertés des paysans, des ouvriers et des Tchèques - Mendel n'a pas, comme eux, accepté la perte de ses droits civiques du fait de son entrée au monastère, perte dont il n'a pris conscience «qu'après des années au prix de tristes expériences ». On peut dès lors comprendre qu'à la faveur des circonstances révolutionnaires il espère rentrer en leur possession autant que jouir des droits nouveaux du citoyen. Ce statut insupportable du moine régulier n'est cependant pas universel puisqu'une solution a été trouvée ailleurs :

3. «En France, le monastère est considéré comme une libre association où le citoyen n'a pas le droit d'être dissous dans le moine, son libre droit de citoyen est à chaque instant reconnu et protégé par l'État comme intangible et au-dessus du contrat privé que constitue son lien avec l'ordre. » L'amertume de Mendel n'est pas récente. Dès ses années de noviciat, l'isolement et le conditionnement imposés aux jeunes clercs derrière les murs du monastère lui avaient été pénibles, souvenirs qui contribuent à sa violence contre l'institution monastique. Cette acrimonie s'exprime dans le paragraphe suivant:

4. «En examinant de plus près la signification ecclésiale et sociale des monastères autrichiens au Xix siècle, on est obligé de reconnaître, en tant qu'observateur impartial, que ces asiles et instituts de l'amour chrétien ne sont moralement rien d'autre que des séminaires forcés; ils sont purement et simplement tombés au rôle d'institution d'assistance pour jeunes gens pauvres et aveuglés. Il faut d'ailleurs avouer que l'isolement forcé de la population, le retrait du cercle familial, le repliement sur soi-même, une éducation déficiente et tendancieuse, la différenciation voyante dans le vêtement, l'attitude et les gestes, l'obéissance absolue révèlent le coup de grâce porté au citoyen dans le moine et représentent l'état du plus profond abaissement. »

Mendel se rebelle d'autant plus contre le destin qui l'attend que la privation de droits civiques s'accompagne d'une dévalorisation sociale du moine aux yeux du monde tant laïc que religieux :

5. «Puisque le droit de libre citoyenneté est volé à l'ensemble du clergé régulier, puisque celui-ci ne compte que comme outil aveugle de la hiérarchie et que l'État a donné son approbation par son droit civil, de ce fait, ce clergé n'a pu bientôt apparaître à la part intelligente du peuple que comme un membre mort de la société, dépourvu de signification; et c'est pourquoi, encore aujourd'hui, les moines comptent pour des zéros dépourvus de droits, sans volonté personnelle, exclus du grand livre d'or des citoyens libres et dignes! Par suite, ils sont exclus de toutes les élections régionales ou nationales, comme celle du Parlement allemand, car la capacité active et passive d'élire tout organisme représentatif leur a été déniée, alors qu'elle est reconnue au travailleur le plus démuni. »

En cette période de recherche frénétique de droits et de liberté, Mendel a découvert avec amertume que les moines sont exclus de toute représentation électorale. Or, d'avril à juin 1848, se déploya une intense activité pour élire les représentants: Parlement allemand à Francfort qui s'ouvre le 18 mai; Diète morave à Brünn le 31 mai ; Congrès slave à Prague début juin et enfin Assemblée constituante impériale le 22 juillet. Cette période riche en activités électorales a été l'occasion pour les jeunes moines de découvrir les nouveaux droits des citoyens, tout en constatant qu'euxmêmes en étaient exclus.

La pétition se termine par une ultime exhortation en faveur des droits civiques et la supplique pour le droit d'enseigner: 6. "Dans la grande Autriche constitutionnelle, dans cet État de citoyens qui se dresse, libre, devant les peuples de l'Europe, comment pourrait-il rester des esclaves exposés au mépris, à la dérision et à la raillerie des peuples libres et civilisés du monde? Ce serait un pilori pour l'Autriche - comparé à l'édifice colossal et structuré de sa Constitution - d'avaliser ces prisons de la citoyenneté, ces tombes de la liberté constitutionnelle. Certes, bientôt l'enterré vivant qui occupe sa cellule sera appelé à la résurrection et à l'activité de citoyen par les puissantes trompettes du premier Parlement autrichien. Dans cette perspective, les professeurs et chargés d'âmes soussignés, prêtres de l'abbaye des Augustins Saint-Thomas à Alt-Brünn, se permettent d'exprimer à la Haute Assemblée de l'Empire la juste requête de la reconnaissance de leur droit constitutionnel de citoyens, avec la demande qu'il leur soit permis, dans la mesure de leur aptitude et du mérite acquis par le passé, de consacrer toute leur énergie spirituelle entièrement à l'enseignement public, entièrement à la libre, une et indivisible citoyenneté. Avec un profond sentiment d'honneur, ils s'assignent comme tâche de leur vie entière la promotion de la Science et de l'Humanité, conformément à l'esprit de progrès constitutionnel.

Brünn, le 8 Août 1848. 
Fr Mattheus Klacel, ancien Professeur de Philosophie. Dr Philipp Gabriel, Professeur de Mathématiques à Brünn, Directeur de l'Institut comtal de Thurn. Josef Lindenthal, Vicaire auprès de la paroisse d'Alt-Brünn. Chrysostomus Cyganek, Candidat professeur. Benedict Fogler, Professeur de Langue et de Littérature françaises, Professeur certifié d'Italien. Gregor Mendel, Vicaire, Candidat professeur $\gg$.

Dans ce texte, le moine Mendel exprime toute la frustration qu'il ressent à la perspective de vivre dorénavant sans droits civiques ni juridiques et d'avoir à subir la dévalorisation sociale de son état: dans le monde laïc, il se refuse à être un «membre mort de la société ». II critique l'institution monastique qu'il traite de «séminaires forcés», refuse de lui devoir «une obéissance absolue», en dénonce les rapports abusifs avec ses membres. Mendel fait l'aveu qu'en entrant au monastère, avec les «autres jeunes gens pauvres et aveuglés», il ignorait tout de la condition du moine et exprime son ressentiment car «malheureusement il n'en a saisi la signification... qu'après des années, au prix de tristes expériences ».

S'il n'avait pas été ordonné prêtre prématurément au cours de l'été 1847, apprenant qu'il n'était plus question pour lui de devenir professeur par la voie religieuse, Mendel aurait pu quitter le monastère à la faveur de la révolution, participer aux élections des représentants, et peut-être même devenir l'un d'entre eux - comme le paysan Kudlich - et surtout exister en tant que citoyen. Cependant, malgré son immense déception, Mendel ne remet pas en cause son appartenance religieuse; il est prêtre et le restera: mais il lutte pour obtenir les droits qui lui redonneront sa dignité. Ainsi, contrairement à d'autres historiens $[4,5]$, nous sommes d'avis que non seulement Mendel avait saisi pleinement toutes les implications des termes de la pétition, mais qu'il en était l'instigateur principal, voire l'auteur selon toute vraisemblance.

\section{La pétition, lettre morte}

L'Assemblée constituante impériale vota, le 7 septembre 1848, le projet présenté par Hans Kudlich, qui abrogeait définitivement les liens de servitude dans tout l'Empire, mais, les travaux sur la constitution ne progressant que très lentement, l'agitation révolutionnaire reprit à Vienne en octobre 1848, menée par les ouvriers. Elle fut réprimée par l'armée dès la fin du mois. En décembre 48, l'empereur Ferdinand abdiqua et François-Joseph lui succéda avec la ferme intention de reprendre les pouvoirs abandonnés par son prédécesseur. Sans avoir changé le statut juridique des moines, le Parlement fut dissous en mars 1849, tandis qu'une vague de néo-absolutisme et de néo-cléricalisme déferlait sur le pays.
Mendel, resté curé d'Alt-Brünn et chapelain de l'hôpital voisin, tomba gravement malade en janvier 1849, à la suite d'une infection probablement contractée au chevet des malades [6] ou lors des autopsies auxquelles il participait [7]. C'est seulement à cette époque, que la pétition fut assignée à la Commission aux affaires religieuses de l'Assemblée constituante, devenant ainsi publique et officiellement connue de l'évêque de Brünn, avec tout le retentissement prévisible au cours d'une telle période de réaction. Mendel fut toutefois nommé professeur adjoint à Znaïm, en septembre 1849, probablement à l'insu de l'évêque [6]. Après la tourmente, la hiérarchie ecclésiastique séculière locale se manifesta clairement en faveur de l'absolutisme. Le comte Schaffgotsche, évêque de Brünn (1841-1870), disposant pour justifier son action d'un document public, tiendra dorénavant Mendel sous surveillance, comme en témoigne le rapport de police de 1849 sur ses faits et gestes à Znaïm, signé par tous ses collègues d'enseignement [1]. Soumis à cette surveillance qui ne faillira pas, Mendel sera amené à masquer ses activités et adoptera en toutes circonstances un comportement discret que ses biographes ont interprété comme une tendance à l'introversion [1, 2]. Mendel va demeurer un «enterré vivant qui occupe sa cellule », il ne sera pas «appelé à la résurrection et à l'activité de citoyen par les puissantes trompettes du premier Parlement autrichien », mais il restera toute sa vie fidèle à ses idées radicales. L'esprit novateur dont Mendel a fait preuve en partageant les aspirations révolutionnaires de son temps a été bridé du fait de son appartenance à un ordre religieux, qui néanmoins lui fournira les conditions favorables à l'éclosion de sa pensée créatrice. $\diamond$

\section{SUMMARY}

1848: Gregor Mendel, the monk who wanted to be a citizen

This article proposes a previously unpublished French translation of a petition, in German, addressed by six Augustinian friars to the Constitutional Parliament of Vienna in the revolutionary year 1848 . The petition states that members of religious orders are deprived of civil rights and demands that they be given citizenship; it also contains a bitter attack on the monastic institution. We suggest that Mendel was the author of this text, which he signed and actually hand-wrote. $\diamond$

\section{RÉFÉRENCES}

1. Iltis H. Gregor Mendel. Leben, Werk und Wirkung. Berlin : Springer, 1924. Traduction anglaise: Life of Mendel. London: Allen and Unwin, 1932. $2^{e}$ ed. New York : Hafner, 1965.

2. Orel V. Gregor Mendel, the first geneticist. Oxford : Oxford University Press, 1996.

3. Weiling F. J.G. Mendel und die Eingabe der capitularen des Stiftes St-Thomas an den Österreichischen Reichstag vom 8. August 1848. Folia Mendeliana 1998/1999; 33/34: 5-10.

4. Orel V, Armogathe JR. Mendel, un inconnu célébre. Paris : Belin, 1985.

5. Orel V, Verbig A. Mendel's involvement in the plea for freedom on teaching in the revolutionary year of 1848 . Folia Mendeliana $1984 ; 19: 223-33$.

6. Nivet C. Une maladie énigmatique dans la vie de Gregor Mendel. Med Sci (Paris) 2004 ; $20: 1050-3$.

7. Coleman W. Ferdinand Schindler letters to William Bateson, 1902-1909. Folia Mendeliana $1967 ; 2: 9-17$.
TIRÉS À PART

C. Nivet 